\title{
On [p, q]-order of Solutions of Higher Order Complex Linear Differential Equations in an Angular Domain of Unit Disc
}

\author{
Jianren Long ${ }^{1,2, *}$ \\ ${ }^{1}$ School of Computer Science and School of Science, Beijing University of Posts \\ and Telecommunications, Beijing, 100876, P. R. China. \\ 2 School of Mathematical Science, Guizhou Normal University, 550001, Guiyang, \\ P.R. China.
}

Received May 18, 2016; Accepted January 25, 2017

\begin{abstract}
We study the growth of solutions of higher order complex linear differential equations in an angular domain of the unit disc instead of the whole unit disc. Some estimations of $[p, q]$-order of solutions of the higher order differential equations in an angular domain are found in this paper.
\end{abstract}

AMS subject classifications: 34M10, 30D35

Key words: Complex differential equation, analytic function, $[p, q]$-order, angular domain, unit disc.

\section{Introduction and main results}

For a function $f$ meromorphic in the unit disc $\Delta=\{z:|z|<1\}$, the order of growth is given by

$$
\rho(f)=\limsup _{r \rightarrow 1^{-}} \frac{\log ^{+} T(r, f)}{\log \frac{1}{1-r}} .
$$

If $f$ is an analytic function in $\Delta$, then the order of growth of $f$ is often given by

$$
\rho_{M}(f)=\limsup _{r \rightarrow 1^{-}} \frac{\log ^{+} \log ^{+} M(r, f)}{\log \frac{1}{1-r}},
$$

where

$$
M(r, f)=\max _{\substack{|z||r| \\ z \in \Delta}}|f(z)|, \quad \log ^{+} x=\max \{\log x, 0\}
$$

*Corresponding author. Email address: longjianren2004@163.com, jrlong@gznu.edu.cn (J.R.Long) 
It follows from the following inequality in [20, Theorem V.13]

$$
T(r, f) \leq \log ^{+} M(r, f) \leq \frac{1+3 r}{1-r} T\left(\frac{1+r}{2}, f\right), \quad r \in(0,1),
$$

that

$$
\rho(f) \leq \rho_{M}(f) \leq \rho(f)+1 .
$$

It is possible that there exists $f$ such that $\rho(f) \neq \rho_{M}(f)$; for example, $f(z)=\exp \left\{\left(\frac{1}{1-z}\right)^{\lambda}\right\}$ satisfies $\rho(f)=\lambda-1$ and $\rho_{M}(f)=\lambda$, where $\lambda>1$ is a constant, which can be found in [20, p. 205].

In order to state our results, some notations are needed. For any $r \in(0, \infty), \exp _{1} r=$ $\exp r, \exp _{n+1} r=\exp \left(\exp _{n} r\right), \log _{1} r=\log r, \log _{n+1} r=\log \left(\log _{n} r\right), n \geq 1$ is integer. $\exp _{0}(r)=$ $r=\log _{0} r, \exp _{-1} r=\log _{1} r$. Second, we recall some definitions.

Definition 1.1 ([10]). For $f$ meromorphic in $\Delta$, set

$$
D(f)=\limsup _{r \rightarrow 1^{-}} \frac{T(r, f)}{\log \frac{1}{1-r}} .
$$

If $D(f)=\infty$, we say that $f$ is admissible. If $D(f)<\infty$, we say that $f$ is non-admissible.

For the function of fast growth in $\Delta$, we also need the definition of iterated $p$-order, which can be found in [4].

Definition 1.2. Let $f$ be a meromorphic function in $\Delta$. Then

$$
\rho_{p}(f)=\limsup _{r \rightarrow 1^{-}} \frac{\log _{p}^{+} T(r, f)}{\log \frac{1}{1-r}},
$$

where $p \geq 1$ is integer. If $f$ is an analytic function in $\Delta$, then the iterated $p$-order is also given by

$$
\rho_{M, p}(f)=\limsup _{r \rightarrow 1^{-}} \frac{\log _{p+1}^{+} M(r, f)}{\log \frac{1}{1-r}} .
$$

Obviously, $\rho_{1}(f) \leq \rho_{M, 1}(f) \leq \rho_{1}(f)+1$ for any analytic functions in $\Delta$. However, it follows from [20, Theorem V.13] that $\rho_{p}(f)=\rho_{M, p}(f)$ for $p \geq 2$. In general, $\rho_{2}(f)$ or $\rho_{M, 2}(f)$ are called hyper-order of $f$ in $\Delta$. In this paper, we assume that the reader is familiar with the fundamental results and standard notation of the Nevanlinna's theory of meromorphic functions in $\Delta$, see [15] and [25] for more details.

Definition $1.3([2,3])$. Let $1 \leq q \leq p$ or $2 \leq q=p+1$, and $f$ be a meromorphic function in $\Delta$. Then the $[p, q]$-order of $f$ is defined as

$$
\rho_{[p, q]}(f)=\limsup _{r \rightarrow 1^{-}} \frac{\log _{p}^{+} T(r, f)}{\log _{q} \frac{1}{1-r}} .
$$


For an analytic function $f$ in $\Delta$, we also define

$$
\rho_{M,[p, q]}(f)=\limsup _{r \rightarrow 1^{-}} \frac{\log _{p+1}^{+} M(r, f)}{\log _{q} \frac{1}{1-r}} .
$$

For the $[p, q]$-order of analytic function $f$ in $\Delta$, we have the following conclusion.

Proposition 1.1 ([21]). Let $f$ be an analytic function of $[p, q]$-order in $\Delta$. Then the following statements hold:

(1) If $p=q=1$, then $\rho(f) \leq \rho_{M}(f) \leq \rho(f)+1$.

(2) If $p=q \geq 2$ and $\rho_{[p, q]}(f)<1$, then $\rho_{[p, q]}(f) \leq \rho_{M,[p, q]}(f) \leq 1$.

(3) If $p=q \geq 2$ and $\rho_{[p, q]}(f) \geq 1$; or $p>q \geq 1$, then $\rho_{[p, q]}(f)=\rho_{M,[p, q]}(f)$.

(4) If $p \geq 1$ and $\rho_{[p, p+1]}(f)>1$, then $D(f)=\infty$; If $\rho_{[p, p+1]}(f)<1$, then $D(f)=0$.

It is always interested in studying the growth of solutions of linear differential equations in the unit disc by using the Nevanlinna's theory of meromorphic functions. The analysis of slowly growing solutions has been studied in $[7,10,11,13,14,18]$. Fast growth of solutions are considered in $[1,5,6,10,12,16]$. There are a few results in studying the growth of solutions of differential equations in an angular domain. One of our main purpose of this paper is to investigate the properties of solutions in an angular domain of the differential equation

$$
A_{k}(z) f^{(k)}+A_{k-1}(z) f^{(k-1)}+\cdots+A_{1}(z) f^{\prime}+A_{0}(z) f=0,
$$

where $A_{0}(z) \not \equiv 0, A_{1}(z), \ldots, A_{k}(z)$ are analytic functions in $\Delta$.

In 2000, Heittokangas studied the growth of solutions of second order linear differential equations and obtained the following result.

Theorem 1.1. ([10]) Suppose that $A_{0}(z), A_{1}(z)$ are analytic functions in $\Delta$ satisfying one of the following conditions.

(1) $\rho\left(A_{1}\right)<\rho\left(A_{0}\right)$;

(2) $A_{0}(z)$ is admissible while $A_{1}(z)$ is non-admissible.

Then all non-trivial solutions of the equation

$$
f^{\prime \prime}+A_{1}(z) f^{\prime}+A_{0}(z) f=0
$$

are of infinite order.

In 2002, Chen generalized Theorem 1.2 to consider arbitrary order equation (1.1). The result is stated as follows. 
Theorem 1.2. ([6]) Suppose that $A_{0}(z), A_{1}(z), \ldots, A_{k}(z)$ are analytic functions in $\Delta$ satisfying one of the following conditions.

(1) $\max _{1 \leq j \leq k} \rho\left(A_{j}\right)<\rho\left(A_{0}\right)$;

(2) $A_{j}(z)$ is non-admissible while $A_{0}$ is admissible, where $j=1,2, \ldots, k$.

Then all non-trivial solutions of the equation (1.1) are of infinite order.

In 1994, Wu [24] studied the growth of solutions of the equation (1.2) in an angular domain by using the Nevanlinna' theory in an angular domain (see [9, Chapters 1 and 3]), and obtained the following result.

Theorem 1.3. Let $A_{0}(z)$ and $A_{1}(z)$ be analytic in $\bar{\Omega}_{\mathbb{C}}(\alpha, \beta)=\{z: \alpha \leq \arg z \leq \beta\}$, where $0 \leq \alpha<$ $\beta \leq 2 \pi$. If for any $l>0$, the measure of

$$
\left\{\theta: \alpha<\theta<\beta, \liminf _{r \rightarrow \infty} \frac{\left(\left|A_{1}\left(r e^{i \theta}\right)\right|+1\right) r^{l}}{\left|A_{0}\left(r e^{i \theta}\right)\right|}=0\right\}
$$

is bigger than zero, then any non-trivial solutions $f$ of $(1.2)$ satisfies $\rho_{\alpha, \beta}(f)=\infty$.

Remark 1.1. The order $\rho_{\alpha, \beta}$ in Theorem 1.4 is defined by

$$
\rho_{\alpha, \beta}(f)=\limsup _{r \rightarrow \infty} \frac{\log ^{+} T_{0}\left(r, \Omega_{\mathbb{C}}(\alpha, \beta), f\right)}{\log r},
$$

where $T_{0}\left(r, \Omega_{\mathbb{C}}(\alpha, \beta), f\right)$ is Ahlfors-Shimizu characteristic in the angular domain $\Omega_{\mathbb{C}}(\alpha, \beta)=$ $\{z: \alpha<\arg z<\beta\}$, see [9] for more details.

In 2009, Xu and Yi [22] generalized Theorem 1.4 to the case of arbitrary order linear differential equation (1.1). The properties of solutions of linear differential equations with analytic coefficients in an angular domain of the whole complex plane are showed in Theorem 1.4 and [22, Theorem 1]. It is natural to ask: What can we say for the growth of solutions of (1.1) with analytic coefficients in an angular domain of the unit disc? We will investigate the problem in this paper. In order to state our results, we also need the following notations. For $0 \leq \alpha<\beta \leq 2 \pi, r>0$, and any given $\varepsilon \in\left(0, \frac{\beta-\alpha}{2}\right)$, set

$$
\begin{aligned}
& \Omega(\alpha, \beta)=\{z: \alpha<\arg z<\beta,|z|<1\}, \\
& \bar{\Omega}(\alpha, \beta)=\{z: \alpha \leq \arg z \leq \beta,|z|<1\}, \\
& \Omega_{\varepsilon}=\{z: \alpha+\varepsilon<\arg z<\beta-\varepsilon,|z|<1\}, \\
& \Omega(r)=\Omega(\alpha, \beta) \cap\{z: 0<|z|<r<1\} .
\end{aligned}
$$


To measure the growth of meromorphic functions in $\Omega(\alpha, \beta)$, we need recall the AhlforsShimizu characteristic in $\Omega(\alpha, \beta)$ (see [20]). In order to state shortly we use $\Omega$ denotes $\Omega(\alpha, \beta)$ in the following statements. Let $f$ ba a meromorphic function in $\bar{\Omega}$. Define

$$
\begin{aligned}
& S(r, \Omega, f)=\frac{1}{\pi} \iint_{\Omega(r)}\left(\frac{\left|f^{\prime}(z)\right|}{1+|f(z)|^{2}}\right)^{2} d \sigma, \\
& T_{0}(r, \Omega, f)=\int_{0}^{r} \frac{S(t, \Omega, f)}{t} d t,
\end{aligned}
$$

where $d \sigma=r d r d \theta$ for $z=r e^{i \theta}$.

Definition $1.4([17,23])$. Let $f$ be a meromorphic function in $\Omega$. Then the order of growth of $f$ is defined by

$$
\rho_{\Omega}(f)=\limsup _{r \rightarrow 1^{-}} \frac{\log ^{+} T_{0}(r, \Omega, f)}{\log \frac{1}{1-r}} .
$$

For fast growing of meromorphic functions in $\Omega$, we use the iterated $p$-order to measure its growing, which is found in [17].

Definition 1.5. Let $f$ be a meromorphic function in $\Omega$. Then

$$
\rho_{\Omega, p}(f)=\limsup _{r \rightarrow 1^{-}} \frac{\log _{p}^{+} T_{0}(r, \Omega, f)}{\log \frac{1}{1-r}} .
$$

To precise measure the growth of meromorphic function in $\Omega$, similarly the case of unit disc, we define $[p, q]$-order.

Definition 1.6. Let $1 \leq q \leq p$, and $f$ be a meromorphic function in $\Omega$. Then the $[p, q]$-order of $f$ is defined as

$$
\rho_{\Omega,[p, q]}(f)=\limsup _{r \rightarrow 1^{-}} \frac{\log _{p}^{+} T_{0}(r, \Omega, f)}{\log _{q} \frac{1}{1-r}} .
$$

We remark that the definitions of $\rho_{\Omega}(f), \rho_{\Omega, p}(f)$ and $\rho_{\Omega,[p, q]}(f)$ of a meromorphic function $f$ in $\Omega$ are reasonable, because

$$
T_{0}(r, \mathbb{C}, f)= \begin{cases}T(r, f)+O(1), & 0<r<1 \\ T(r, f)+O(\log r), & 0<r<\infty\end{cases}
$$

where $\mathbb{C}$ denotes whole complex plane.

In this paper, we mainly obtain the following results by using the similar way of [23].

Theorem 1.4. Let $p \geq q \geq 1$ and $A_{0}(z), A_{1}(z), \ldots, A_{k}(z)$ be analytic functions in $\Omega=\{z: \alpha<$ $\arg z<\beta,|z|<1\}$. If

$$
\max _{1 \leq j \leq k}\left\{\rho_{\Omega,[p, q]}\left(A_{j}\right)\right\}<\rho_{\Omega_{\varepsilon},[p, q]}\left(A_{0}\right),
$$


then every non-trivial solution $f$ of (1.1) satisfies

$$
\rho_{\Omega,[p+1, q]}(f) \geq \rho_{\Omega_{\varepsilon},[p, q]}\left(A_{0}\right) .
$$

To state the second result, we also need the following notations. For $H \subset[0,1)$, the upper and lower densities of $H$ are given, which can be found in [5], see also [3], by

$$
\overline{\operatorname{dens}}(H)=\limsup _{r \rightarrow 1^{-}} \frac{\mathrm{m}(H \cap[0, r))}{\mathrm{m}([0, r))}, \quad \underline{\operatorname{dens}}(H)=\liminf _{r \rightarrow 1^{-}} \frac{\mathrm{m}(H \cap[0, r))}{\mathrm{m}([0, r))}
$$

respectively, where $\mathrm{m}(G)=\int_{G} \frac{d t}{1-t}$ for $G \subset[0,1)$.

Theorem 1.5. Let $p \geq q \geq 1$ and $F$ be a set of complex numbers satisfying $\overline{\operatorname{dens}}(\{|z|: z \in F \subset$ $\Omega\})>0$, and let $A_{0}(z), A_{1}(z), \ldots, A_{k}(z)$ be analytic functions in $\Omega=\{z: \alpha<\arg z<\beta,|z|<1\}$ such that for some real constants $0 \leq \gamma<\lambda$, we have

$$
\begin{aligned}
& T_{0}\left(r, \Omega_{\varepsilon}, A_{0}\right) \geq \exp _{p}\left\{\lambda \log _{q}\left(\frac{1}{1-|z|}\right)\right\}, \\
& T_{0}\left(r, \Omega, A_{j}\right) \leq \exp _{p}\left\{\gamma \log _{q}\left(\frac{1}{1-|z|}\right)\right\}, \quad j=1, \ldots, k,
\end{aligned}
$$

as $|z|=r \rightarrow 1^{-}$for $z \in F$. Then every non-trivial solution $f$ of $(1.1)$ satisfies $\rho_{\Omega,[p, q]}(f)=\infty$ and $\rho_{\Omega,[p+1, q]}(f) \geq \lambda$.

\section{Auxiliary results}

In this section, we give some auxiliary results for the proof of our theorems. The proof of the following lemma can be found in [19] plays an important role in proving our results.

Lemma 2.1. Let

$$
\zeta(z)=\frac{\left(z e^{-i \theta_{0}}\right)^{\frac{\pi}{\delta}}+2\left(z e^{-i \theta_{0}}\right)^{\frac{\pi}{2 \delta}}-1}{\left(z e^{-i \theta_{0}}\right)^{\frac{\pi}{\delta}}-2\left(z e^{-i \theta_{0}}\right)^{\frac{\pi}{2 \delta}}-1}
$$

where $0 \leq \theta_{0}=\frac{\alpha+\beta}{2}<2 \pi, 0<\delta=\frac{\beta-\alpha}{2}<\pi$. Then $\zeta(z)$ is a conformal map of angular domain $\Omega=\{z: \alpha<\arg z<\beta,|z|<1\}$ onto the unit disc $\Delta$. Moreover, for any positive number $\varepsilon<\delta$, the transformation (2.1) satisfies

$$
\begin{aligned}
& \zeta\left(\left\{\frac{1}{2}<|z|<r\right\} \cap\left\{z:\left|\arg z-\theta_{0}\right|<\delta-\varepsilon\right\}\right) \subset\left\{\zeta:|\zeta|<1-\frac{\varepsilon}{2^{\frac{\pi}{2 \delta}+1} \delta}(1-r)\right\}, \\
& \zeta^{-}(\{\zeta:|\zeta|<\rho\}) \subset\left(\left\{z:|z|<1-\frac{\delta}{8 \pi}(1-\rho)\right\} \cap\left\{z:\left|\arg z-\theta_{0}\right|<\delta\right\}\right),
\end{aligned}
$$

where $\rho<1$ is a constant. The inverse transformation of (2.1) is

$$
z(\zeta)=e^{i \theta_{0}}\left[\frac{-(1+\zeta)+\sqrt{2\left(1+\zeta^{2}\right)}}{1-\zeta}\right]^{\frac{2 \delta}{\pi}}
$$


The following lemma due to $\mathrm{Wu}[23$, Lemma 2.2].

Lemma 2.2. Let $f$ be a meromorphic function in $\Omega=\{z: \alpha<\arg z<\beta,|z|<1\}$, where $0 \leq \alpha<$ $\beta<2 \pi$. For any given $\varepsilon \in\left(0, \frac{\beta-\alpha}{2}\right)$, set

$$
\delta=\frac{\beta-\alpha}{2}, \quad b=\frac{\varepsilon}{2^{\frac{\pi}{2 \delta}}+1 \delta} .
$$

Then the following statements hold.

$$
\begin{aligned}
& T_{0}(r, \mathbb{C}, f(z(\zeta))) \leq \frac{16 \pi}{\delta} T_{0}\left(1-\frac{\delta}{8 \pi}(1-r), \Omega, f(z)\right)+O(1), \\
& T_{0}\left(r, \Omega_{\varepsilon}, f(z)\right) \leq \frac{2}{b} T_{0}(1-b(1-r), \mathbb{C}, f(z(\zeta)))+O(1),
\end{aligned}
$$

where $z(\zeta)$ is the inverse transformation of (2.1).

Remark 2.1. Applying the formula $T(r, f)=T_{0}(r, \mathbb{C}, f)+O(1)$, Lemma 2.2 and the definition of $[p, q]$-order, we immediately obtain that

$$
\rho_{[p, q]}(f(z(\zeta))) \leq \rho_{\Omega,[p, q]}(f(z)), \quad \rho_{\Omega_{\varepsilon},[p, q]}(f(z)) \leq \rho_{[p, q]}(f(z(\zeta))) .
$$

The following lemma can be proved by the same method of [8, Lemma 1], see also [23, Lemma 2.3].

Lemma 2.3. Let $f$ be a meromorphic function in $\Omega=\{z: \alpha<\arg z<\beta,|z|<1\}$, where $0 \leq \alpha<$ $\beta<2 \pi$, and $z=z(\zeta)$ be the inverse transformation of (2.1). Set

$$
F(\zeta)=f(z(\zeta)), \quad \psi(\zeta)=f^{(l)}(z(\zeta))
$$

Then

$$
\psi(\zeta)=\sum_{j=1}^{l} \alpha_{j} F^{(j)}(\zeta)
$$

where the coefficients $\alpha_{j}$ are the polynomials (with numerical coefficients) in the variables $V(\zeta)(=$ $\left.\frac{1}{z^{\prime}(\zeta)}\right), V^{\prime}(\zeta), \cdots$. Moreover, we have $T\left(r, \alpha_{j}\right)=O\left(\log \frac{1}{1-r}\right), j=1,2, \ldots, l$. 


\section{Proofs of Theorems}

Proof of Theorem 1.5. Suppose that $f$ is a non-trivial solution of (1.1) in $\Omega$. Applying Lemma 2.3, we have

$$
\begin{aligned}
& \sum_{i=1}^{k} A_{i}(z(\zeta)) f^{(i)}(z(\zeta))+A_{0}(z(\zeta)) f(z(\zeta)) \\
= & \sum_{i=1}^{k} A_{i}(z(\zeta)) \sum_{j=1}^{i} \alpha_{j} F^{(j)}(\zeta)+A_{0}(z(\zeta)) f(z(\zeta)) \\
= & \sum_{j=1}^{k} \alpha_{j} \sum_{i=j}^{k} A_{i}(z(\zeta)) F^{(j)}(\zeta)+A_{0}(z(\zeta)) f(z(\zeta)) .
\end{aligned}
$$

Then $F(\zeta)=f(z(\zeta))$ is a solution of the differential equation

$$
B_{k}(\zeta) F^{(k)}(\zeta)+B_{k-1}(\zeta) F^{(k-1)}(\zeta)+\cdot+B_{0}(\zeta) F(\zeta)=0, \text { in } \Delta,
$$

where

$$
B_{0}(\zeta)=A_{0}(z(\zeta)), \quad B_{j}(\zeta)=\alpha_{j} \sum_{i=j}^{k} A_{i}(z(\zeta)), j=1, \ldots, k,
$$

are analytic in $\Delta$. Since $T\left(r, \alpha_{j}\right)=O\left(\log \frac{1}{1-r}\right), j=1, \ldots, k$, hence it follows from this and the Nevanlinna's theory that

$$
\begin{aligned}
T\left(r, B_{j}\right) & \leq T\left(r, \alpha_{j}\right)+\sum_{i=j}^{k} T\left(r, A_{i}(z(\zeta))\right)+O(1) \\
& =\sum_{i=j}^{k} T_{0}\left(r, \mathbb{C}, A_{i}(z(\zeta))\right)+O\left(\log \frac{1}{1-r}\right), \quad j=1, \ldots, k
\end{aligned}
$$

Applying Lemma 2.2 and our conditions, we get

$$
\begin{aligned}
& \rho_{[p, q]}\left(B_{j}\right) \leq \max _{1 \leq j \leq k} \rho_{[p, q]}\left(A_{j}\right) \leq \max _{1 \leq j \leq k} \rho_{\Omega,[p, q]}\left(A_{j}\right)<\rho_{\Omega_{\varepsilon,}[p, q]}\left(A_{0}\right), \\
& \rho_{[p, q]}\left(B_{0}\right)=\rho_{[p, q]}\left(A_{0}\right) \geq \rho_{\Omega_{\varepsilon},[p, q]}\left(A_{0}\right) .
\end{aligned}
$$

It follows from two inequalities above that

$$
\rho_{[p, q]}\left(B_{j}\right)<\rho_{[p, q]}\left(B_{0}\right), \quad \text { for } j=1, \ldots, k .
$$

By [21, Theorem 2.1], we get $\rho_{[p+1, q]}(F) \geq \rho_{[p, q]}\left(B_{0}\right)$ for all non-trivial solutions $F$ of (3.1). It follows from this and (2.5) that

$$
\rho_{\Omega,[p+1, q]}(f) \geq \rho_{[p+1, q]}(f(z(\zeta)))=\rho_{[p+1, q]}(F) \geq \rho_{[p, q]}\left(B_{0}\right) \geq \rho_{\Omega_{\varepsilon}[p, q]}\left(A_{0}\right) .
$$

Thus $\rho_{\Omega,[p+1, q]}(f) \geq \rho_{\Omega_{\varepsilon}[p, q]}\left(A_{0}\right)$ for all non-trivial solutions $f$ of (1.1). This completes the proof. 
Proof of Theorem 1.6. Suppose that $f$ is a non-trivial solution of (1.1) in $\Omega$. By using the similar reasoning of the proof of Theorem 1.5, we get (3.1). By (2.3) and (2.4) in Lemma 2.2, we get

$$
\begin{aligned}
T\left(r, B_{0}\right) & =T\left(r, A_{0}\right)=T_{0}\left(r, \mathbb{C}, A_{0}(z(\zeta))\right)+O(1) \\
& \geq \frac{b}{2} T_{0}\left(1-\frac{1-r}{b}, \Omega_{\varepsilon}, A_{0}\right)+O(1) \\
& \geq \frac{b}{2} \exp _{p}\left\{\lambda \log _{q}\left(\frac{b}{1-r}\right)\right\}=O\left(\exp _{p}\left\{\lambda \log _{q}\left(\frac{1}{1-r}\right)\right\}\right)
\end{aligned}
$$

and for $j=1, \ldots, k$,

$$
\begin{aligned}
T\left(r, B_{j}\right) & \leq \sum_{i=j}^{k} T\left(r, A_{i}(z(\zeta))\right)+O\left(\log \frac{1}{1-r}\right) \\
& =\sum_{i=j}^{k} T_{0}\left(r, \mathbb{C}, A_{i}(z(\zeta))\right)+O\left(\log \frac{1}{1-r}\right) \\
& \leq \frac{16 \pi}{\delta} \sum_{i=j}^{k} T_{0}\left(1-\frac{\delta}{8 \pi}(1-r), \Omega, A_{i}\right)+O\left(\log \frac{1}{1-r}\right) \\
& \leq \frac{16 \pi}{\delta} \exp _{p}\left\{\gamma \log _{q}\left(\frac{1}{\frac{\delta}{8 \pi}(1-r)}\right)\right\}=O\left(\exp _{p}\left\{\gamma \log _{q}\left(\frac{1}{1-r}\right)\right\}\right),
\end{aligned}
$$

as $|z|=r \rightarrow 1^{-}$and $z \in G$, where the set $G$ is a set of image of $F$ by transformation (2.1) satisfying $\overline{\operatorname{dens}}\{|\zeta|: \zeta \in G\}>0$.

Applying [3, Theorem 1.1], we get $\rho_{[p, q]}(F)=\infty$ and $\rho_{[p+1, q]}(F) \geq \lambda$ for all non-trivial solutions $F$ of (3.1). It follows from this and (2.5) that

$$
\rho_{\Omega,[p, q]}(f)=\infty, \quad \rho_{\Omega,[p+1, q]}(f) \geq \lambda
$$

for all non-trivial solutions $f$ of (1.1). This completes the proof.

\section{Acknowledgments}

This research is supported in part by the National Natural Science Foundation of China (11501142, 11571288), and the Foundation of Science and Technology of Guizhou Province of China ([2015]2112).

\section{References}

[1] D. Benbourenane, L.R. Sons. On global solutions of complex differential equations in the unit disc. Complex Var. Elliptic Equa., 2004, 49: 913-925. 
[2] B. Belaïdi. Growth of solutions to linear equations with analytic coefficients of $[p, q]$-order in the unit disc. Electron. J. Diff. Equa., 2011, 156: 1-11.

[3] B. Belaïdi. On the $[p, q]$-order of analytic solutions of linear differential equations in the unit disc. Novi Sad J. Math., 2012, 42(1): 117-129.

[4] T.B. Cao, H.X. Yi. The growth of solutions linear differential equations with coefficients of iterated order in the unit disc. J. Math. Anal. Appl., 2006, 319: 278-294.

[5] T.B. Cao. The growth, oscillation and fixed points of solutions of complex linear differential equations in the unit disc. J. Math. Anal. Appl., 2009, 352: 739-748.

[6] Z.X. Chen. The properties of solutions of a class of differential equations in the unit disc. J. Jiangxi Norm. Univer., 2002, 26(3): 189-190.

[7] Z.X. Chen, K.H. Shon. The growth of solutions of differential equations with coefficients of small growth in the disc. J. Math. Anal. Appl., 2004, 297: 285-304.

[8] A. Edrei. Meromorphic functions with three radially distributed values. Trans. Amer. Math. Soc., 1955, 78: 276-293.

[9] A.A. Gol'dberg, I.V. Ostrovshii. Value Distribution of Meromorphic Functions. Translations of Mathematical Monographs, 236, AMS, Providence, RI, 2008.

[10] J. Heittokangas. On complex differential equations in the unit disc. Ann. Acad. Sci. Fenn. Math. Diss., 2000, 122: 1-54.

[11] J. Heittokangas. Blaschke-oscillatory equations of the form $f^{\prime \prime}+A(z) f=0$. J. Math. Anal. Appl., 2006, 318: 120-133.

[12] J. Heittokangas, R. Korhonen, J. Rättyä. Fast growing solutions of linear differential equations in the unit disc. Results Math., 2006, 49: 265-278.

[13] J. Heittokangas, R. Korhonen, J. Rättyä. Linear differential equations with coefficients in weighted Bergman and Hardy spaces. Trans. Amer. Math. Soc., 2007. 360(2): 1035-1055.

[14] J. Heittokangas, R. Korhonen, J. Rättyä. Linear differential equations with solutions in the Dirichlet type subspace of Hardy spaces. Nagoya Math. J., 2007, 187: 91-113.

[15] W.K. Hayman. Meromorphic Functions. Clarendon Press, Oxford, 1964.

[16] J.R. Long. Growth of solutions of higher order complex linear differential equations in an angular domain. J. Math. (Wuhan), 2015 ,35(6): 1533-1540.

[17] J.R. Long. Growth of solutions of higher order complex linear differential equations in an angular domain of unit disc. J. Math. Study, 2015, 48(3): 306-314.

[18] Chr. Pommenrenke. On the mean grwoth of solutions of complex linear differential equations in the disk. Complex Var. Elliptic Equa., 1982, 1(1): 23-38.

[19] D.C. Sun, J.R. Yu. On the distribution of random Dirichlet series (II). Chin. Ann. Math., 1990, 11B: 33-44.

[20] M. Tsuji. Potential Theory in Modern Function Theory. Chelsea, New York, 1975, reprint of the 1959 edition.

[21] J. Tu, H.X. Huang. Complex oscillation of linear differential equations with analytic coefficients of $[p, q]$-order in the unit disc. Comput. Methods Funct., 2015, 15(2): 225-246.

[22] J.F. Xu, H.X. Yi. Solutions of higher order linear differential equations in an angle. Appl. Math. Letter, 2009, 22(4): 484-489.

[23] N. Wu. On the growth order of solutions of linear differential equations in a sector of the unit disk. Results Math., 2014, 65: 57-66.

[24] S.J. Wu. On the growth of solutions of second order linear differential equations in an angle. Complex Var., 1994, 24: 241-248.

[25] G.H. Zhang. Theory of Entire and Meromorphic Functions-Deficient and Asymptotic Values and Singular Directions. Springer-Verlag, Berlin, 1993. 